\title{
Turbulent dispersion in cloud-topped boundary layers
}

\author{
R. A. Verzijlbergh ${ }^{1}$, H. J. J. Jonker ${ }^{1}$, T. Heus ${ }^{1, *}$, and J. Vilà-Guerau de Arellano ${ }^{2}$ \\ ${ }^{1}$ Department of Multi-Scale Physics, Delft University of Technology, Delft, The Netherlands \\ ${ }^{2}$ Meteorology and Air Quality Section, Wageningen University, The Netherlands \\ *current affiliation: Royal Netherlands Meteorological Institute, De Bilt, The Netherlands
}

Received: 22 September 2008 - Published in Atmos. Chem. Phys. Discuss.: 18 November 2008

Revised: 5 February 2009 - Accepted: 9 February 2009 - Published: 18 February 2009

\begin{abstract}
Compared to dry boundary layers, dispersion in cloud-topped boundary layers has received less attention. In this LES based numerical study we investigate the dispersion of a passive tracer in the form of Lagrangian particles for four kinds of atmospheric boundary layers: 1) a dry convective boundary layer (for reference), 2) a "smoke" cloud boundary layer in which the turbulence is driven by radiative cooling, 3) a stratocumulus topped boundary layer and 4) a shallow cumulus topped boundary layer.

We show that the dispersion characteristics of the smoke cloud boundary layer as well as the stratocumulus situation can be well understood by borrowing concepts from previous studies of dispersion in the dry convective boundary layer. A general result is that the presence of clouds enhances mixing and dispersion - a notion that is not always reflected well in traditional parameterization models, in which clouds usually suppress dispersion by diminishing solar irradiance.

The dispersion characteristics of a cumulus cloud layer turn out to be markedly different from the other three cases and the results can not be explained by only considering the well-known top-hat velocity distribution. To understand the surprising characteristics in the shallow cumulus layer, this case has been examined in more detail by 1) determining the velocity distribution conditioned on the distance to the nearest cloud and 2) accounting for the wavelike behaviour associated with the stratified dry environment.
\end{abstract}

Correspondence to: H. J. J. Jonker (h.j.j.jonker@tudelft.nl)

\section{Introduction}

This paper describes the dispersion of a passive tracer in different types of atmospheric boundary layers with emphasis on the dispersion in cloudy boundary layers. Understanding the diffusion of pollutants in cloudy boundary layers is important for climate, atmospheric chemistry and air quality. Clouds are known to transport pollutants from the boundary layer to higher regions in the atmosphere, a phenomenon referred to as cloud venting (e.g., Cotton, 1995). An intricate coupling exists between particles in the atmosphere and clouds: not only do clouds enhance the upward vertical transport of pollutants (gases, aerosols) they are also strongly influenced by them. The optical properties as well as the lifetime of a cloud are known to depend on the aerosol distribution in the cloud's environment. In turn, both the optical properties of clouds as well as their lifetimes affect the earth's radiation budget and hence global climate.

Chemical processes in the atmosphere are also influenced by clouds. First of all they affect transport of chemical compounds through the atmosphere and enhance turbulent mixing of different species. In addition clouds can alter the photodissociation rates of chemical compounds around them (Vilà-Guerau de Arellano et al., 2005).

Finally, next to the importance of dispersion on climate and atmospheric chemistry, the quality of the air we live in is also affected by meteorological conditions. Predicting ground level concentrations of possibly harmfull substances requires detailed knowledge about the relation between weather conditions and dispersion.

The classical work relating dispersion and turbulence was done by Taylor (1921). This analysis, however, was based on homogeneous turbulence, whereas atmospheric motions are often very complex and characterized by non-homogeneous turbulence. Pasquill (1961) proposed a Gaussian plume model with a vertical dispersion coefficient depending on the

Published by Copernicus Publications on behalf of the European Geosciences Union. 
meteorological circumstances. Basically, the vertical dispersion coefficient is then related to the stability of the atmosphere, which is related to the amount of insolation. In this view, clouds have a damping effect on dispersion in daytime conditions.

The subject of atmospheric dispersion has been further extensively studied in the laboratory, in field experiments and by numerical methods. The pioneering water tank experiments of Willis and Deardorff (1978) demonstrated the effects of the non-homogeneous turbulence of a convective boundary layer (CBL) on the diffusion of particles. They showed, rather surprisingly at the time, that a near-ground release resulted in a quickly rising plume (in terms of the peak concentration), but an elevated release resulted in a descending plume that only rises after impinging on the ground. The water tank results have later been verified by full-scale atmospheric experiments (Briggs, 1993). Lamb (1978) approached the problem numerically and used the velocity fields from Large Eddy Simulations to investigate the dispersion of particles. Among others, Nieuwstadt and de Valk (1987) used the advection of a passive scalar in an LES model to describe the dispersion of both buoyant and non-buoyant plumes in the dry CBL. More recently Dosio and Vilà-Guerau de Arellano (2006) gave a thorough statistical description of dispersion in the dry CBL in an LES based study. By now, it is well understood that the skewed velocity distribution is responsible for the observed descent of the plume maximum for elevated releases of non-buoyant plumes in the CBL.

In contrast with the number of studies on dispersion in the $\mathrm{CBL}$ is the modest number of studies on dispersion in other types of boundary layers, in particular cloudy conditions. Dispersion in the stable boundary layer was studied by e.g. Hunt (1985), Kemp and Thomson (1996) and more recently Weil et al. (2006). The effects of a stratocumulus cloud deck on dispersion in the nocturnal boundary layer have been explored by Sorbjan and Uliasz (1999). Evidence was found that the vertical diffusion of pollutants in a stratocumulus topped boundary layer is non-Gaussian and depends on the location of the source in the boundary layer. Concerning shallow cumulus clouds, some field experiments have demonstrated the effect of cloud venting (e.g., Ching et al., 1988; Angevine, 2005). Vilà-Guerau de Arellano et al. (2005) have shown in a LES study how shallow cumulus enhance vertical transport of pollutants, thereby specifically focussing on the influence on chemical transformations. Weil et al. (1993) used ice-crystals as a tracer to study relative dispersion in an ensemble of cumulus clouds. Chosson et al. (2008) did a numerical study of the dispersion of ship tracks, and the role of stratocumulus and cumulus clouds therin. They focussed mainly on the first few turn-over times of the cloud venting process.

Because a comprehensive study of dispersion in cloudy boundary layers that includes dispersion over longer time scales of pollution within the cloud layer appears to be miss- ing, the objective of this detailed numerical study is to investigate and statistically describe turbulent dispersion in different types of cloudy boundary layers. To this end we perform large eddy simulations together with a Lagrangian particle module. Four types of boundary layers will be considered: 1) the clear convective boundary layer, 2) a boundary layer filled with radiatively cooling smoke, 3) the stratocumulus topped boundary layer and finally 4) the shallow cumulus topped boundary layer. The differences and similarities between these four atmospheric situations offer a unique opportunity to gain more insight in the observed dispersion characteristics. The primary aim of this paper is to study the influence of the clouds on the dispersion. Where necessary, the deeper mechanisms that control the dispersion in and around clouds will be briefely treated. In Sect. 2 we describe the methodology consisting of the numerical setup, the case characteristics and the definition of statistical quantities. Section 3, in which the results are presented and discussed, is divided into two parts: a phenomenological part with a qualitative description of the dispersion characteristics in the different boundary layers is followed by a more quantitative part.

\section{Methodology}

\subsection{LES model and Lagrangian particle dispersion model}

The LES-code used in this research is version 3 of the Dutch Atmospheric LES (DALES3) as described by Cuijpers and Duynkerke (1993). In this study, Lagrangian particles rather than a concentration field of a scalar are used as a representation of the pollutants. To this end, a Lagrangian Particle Dispersion Module (LPDM) as described in Heus et al. (2008) is implemented in the LES. Tri-linear interpolation determines the particle location within each grid cell; the LPDM takes subgrid-scale motion into account through a model that is largely based on the criteria for stochastic Lagrangian models formulated by Thomson (1987). The implementation of these criteria in LES models described in Weil et al. (2004) is followed in the present LPDM. Due to the parallelization of the LES some extra attention was required to handle the case of particles moving from one processor to another. A dynamical list structure in the form of a linked list was used to cope with this issue: a particle moving from, say, the first processor to the second will be deleted from the list of the first and added to the list of the second processor. Every record in the list hence points to a particle and every record can contain as many entries as wished, e.g., positions, velocities, temperature etc.

The use of Lagrangian particles has the advantage of being able to track individual particles in time, thereby allowing the calculation of Lagrangian statistics. Contrary to Nieuwstadt and de Valk (1987), an instantaneous plane source rather than an instantaneous line source is used in this study. We 
can view the plane source of $1024^{2} \approx 10^{6}$ particles homogeneously distributed over the domain in both horizontal directions as an ensemble of 1024 linesources, that are released instantaneously. For every case, 3 simulations are run, with the release height close to the boundary layer height $z_{i}\left(z=0.2 z_{i}\right)$, in the center of the boundary layer $\left(z=0.5 z_{i}\right)$, and near the surface $\left(z=0.2 z_{i}\right)$, respectively. For all cloudy cases, we define the boundary layer height as the height of the maximum gradient in $\theta_{v}$. This definition ensures that the cloud layer is incorporated within the boundary layer. Details about the numerics of the simulations vary between the different cases and will be discussed in the next section.

\subsection{Case descriptions}

Hereafter we describe briefly the four different atmospheric situations that have been under consideration. In particular the velocity distributions (depicted schematically in Fig. 1) are discussed, since they are important for the dispersion characteristics. Numerical values of the case characteristics are listed in Table 1. Figure 2 shows the profiles of the virtual potential temperature flux $\left\langle\overline{w^{\prime} \theta_{v}^{\prime}}\right\rangle$. These profiles give an indication about the dynamics and the structure of the boundary layer.

\subsubsection{Dry convective boundary layer}

The CBL is characterized by a well mixed layer, a strong surface heat flux and a capping inversion. This gives rise to a positively skewed velocity distribution: strong localized updrafts surrounded by moderate compensating downdrafts, as depicted schematically in Fig. 1a. Figure 2a shows the buoyancy flux profile for the CBL. The boundary layer height, averaged over the first hour, was $900 \mathrm{~m}$ high, as given in Table 1, and increased slightly during the simulation. No large scale horizontal wind was imposed. The simulation was run on a grid of $256^{3}$ points, with a horizontal resolution of $\Delta x=\Delta y=25 \mathrm{~m}$ and a vertical of $\Delta z=6 \mathrm{~m}$, resulting in a domain of $6.4 \mathrm{~km} \times 6.4 \mathrm{~km} \times 1.5 \mathrm{~km}$. A timestep of $\Delta t=1 \mathrm{~s}$ and a 5 th order advection scheme (Wicker and Skamarock, 2002) have been used, except for the advection of momentum, for which a second order central-difference scheme has been used. The particles were released after three hours of simulation.

\subsubsection{Smoke cloud boundary layer}

The smoke case used in this study is described in an intercomparison study by Bretherton et al. (1999). The smoke case is particularly useful to gain understanding of the stratocumulus case, for it has similar radiation characteristics, but there are no condensation processes or surface fluxes to additionally drive convection. Making the analogy with the CBL, instead of heating at the bottom (CBL) we have radiative cooling due to the divergence of the radiative flux at the

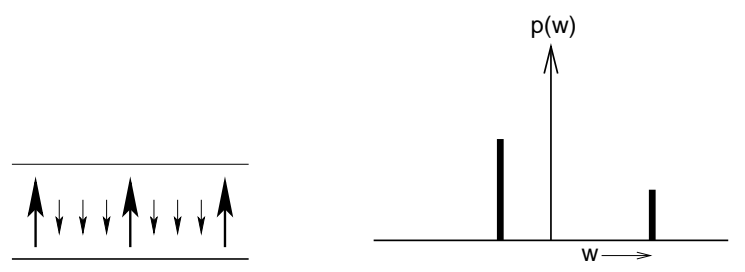

(a) $\mathrm{CBL}$

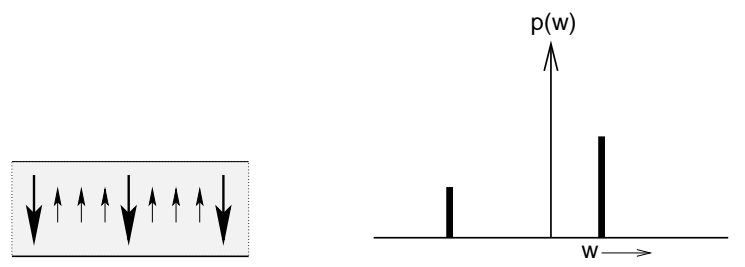

(b) Smoke

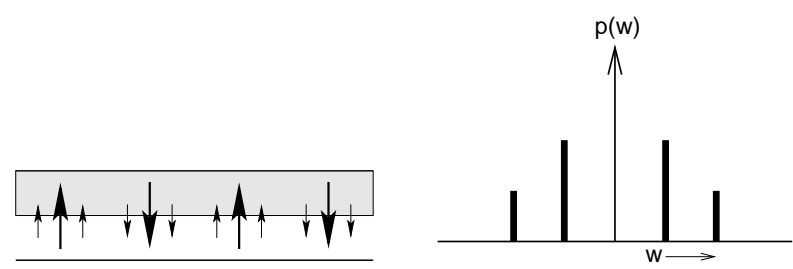

(c) Stratocumulus

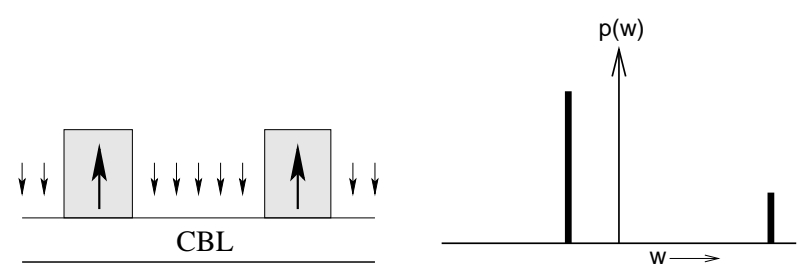

(d) Cumulus

Fig. 1. Conceptual representation of the velocity distributions in the different boundary layers.

top (Smoke). This results in a mirror image of the vertical velocity distribution from the CBL, as depicted in Fig. $1 \mathrm{~b}$

Figure $2 b$ shows the buoyancy flux in the smoke cloud. Next to the absence of a surface heat flux, we also observe entrainment at the top of the smoke cloud.

In the smoke case the domain measured $3.2 \mathrm{~km} \times 3.2 \mathrm{~km} \times 1.2 \mathrm{~km}$. Horizontal and vertical resolutions are $\Delta x=\Delta y=12.5 \mathrm{~m}$ and $\Delta z=6.25 \mathrm{~m}$ and the number of grid points is 256 in the horizontal and 200 in the vertical direction. For the scalar variables the monotonous kappa advection scheme (Hundsdorfer et al., 1995) and for momentum the second order central-differences scheme with a timestep of $\Delta t=0.5 \mathrm{~s}$ have been used. The particles were released after two hours of simulation. 
Table 1. Characteristics of the different cases: the approximate boundary layer height $z_{i}$ at the moment of particle release, the cloud base height $z_{c b}$ at the moment of particle release, the surface moisture and heat fluxes $\left.\overline{\left\langle w^{\prime} q_{t}^{\prime}\right.}\right\rangle_{0}$ and $\left.\overline{\left\langle w^{\prime} \theta_{l}^{\prime}\right.}\right\rangle_{0}$, the convective velocity scale $w_{*}$ as defined in Eq. (2), the characteristic timescale $t_{*}$.

\begin{tabular}{lcccccc}
\hline & $\begin{array}{c}z_{i} \\
{[\mathrm{~m}]}\end{array}$ & $\begin{array}{c}z_{c b} \\
{[\mathrm{~m}]}\end{array}$ & $\begin{array}{c}\left\langle\overline{w^{\prime} q_{t}^{\prime}}\right\rangle_{0} \\
{\left[\mathrm{~kg} \mathrm{~kg}^{-1} \mathrm{~m} \mathrm{~s}^{-1}\right]}\end{array}$ & $\begin{array}{c}\left\langle\overline{w^{\prime} \theta_{l}^{\prime}}\right\rangle_{0} \\
{\left[\mathrm{~K} \mathrm{~m} \mathrm{~s}^{-1}\right]}\end{array}$ & $\begin{array}{c}w_{*} \\
{\left[\mathrm{~m} \mathrm{~s}^{-1}\right]}\end{array}$ & $\begin{array}{c}t_{*} \\
{[\mathrm{~s}]}\end{array}$ \\
\hline dry CBL & 900 & - & 0 & $9.4 \times 10^{-2}$ & 1.42 & 633 \\
smoke & 700 & 0 & 0 & 0 & 0.92 & 760 \\
stratocumulus & 700 & 350 & $1 \times 10^{-5}$ & $1 \times 10^{-2}$ & 0.87 & 804 \\
shallow cumulus & 2000 & 500 & $1.2 \times 10^{-4}$ & $1.5 \times 10^{-2}$ & 1.66 & 1204 \\
\hline
\end{tabular}

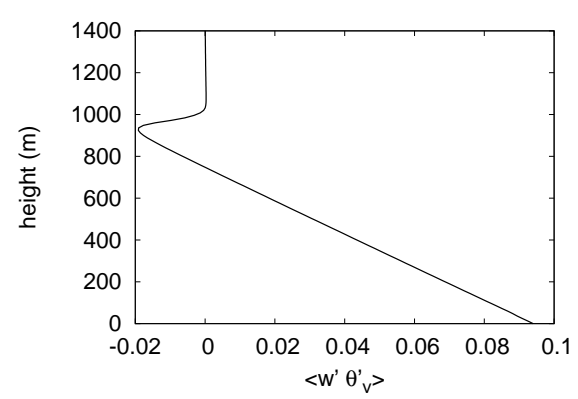

(a) $\mathrm{CBL}$

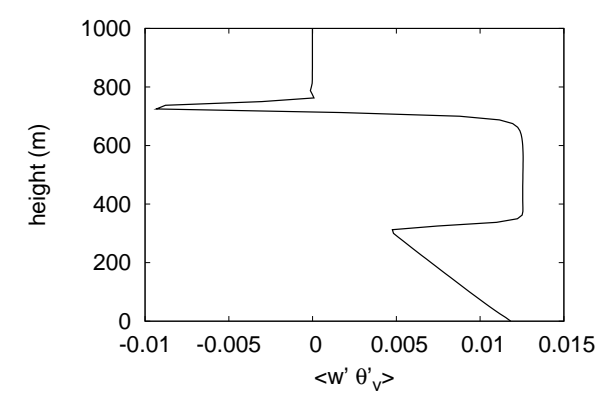

(c) Stratocumulus

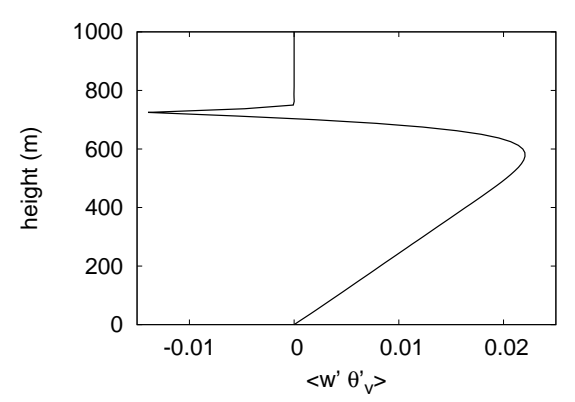

(b) Smoke

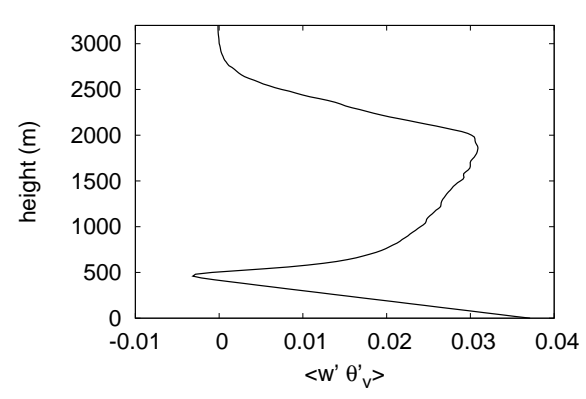

(d) Cumulus

Fig. 2. Virtual potential temperature flux $\left\langle\overline{w^{\prime} \theta_{v}^{\prime}}\right\rangle$ in the four different boundary layers. The profiles are an average over the first hour after particle-release.

\subsubsection{Stratocumulus topped boundary layer}

The stratocumulus case under consideration is the Atlantic Stratocumulus Transition Experiment (ASTEX, de Roode and Duynkerke, 1997). Data from flight 2, A209, has been used. Convection in a stratocumulus topped boundary layer is driven by a combination of processes: radiative cooling at cloud top, surface fluxes of heat and moisture and latent heat release due to condensation. This is the only case that is subject to a mean horizontal wind of $(0.5,-10) \mathrm{m} \mathrm{s}^{-1}$, that is roughly constant throughout the bulk of the domain. In analogy with the previous cases, in terms of the driving mechanisms, the stratocumulus case can be regarded a combination of the CBL and the smoke cloud. This translates into a velocity distribution that is a combination of the $\mathrm{CBL}$ and the smoke case, thus giving rise to a more symmetric velocity distribution, as depicted in Fig. 1c.

Figure $2 \mathrm{c}$ shows the virtual potential temperature flux of the stratocumulus case. We already stated that the stratocumulus case is a combination of the CBL and the smoke case, but here we specify that it is especially in the cloud layer (starting at approximately $350 \mathrm{~m}$ ) that the smoke cloud characteristics are found. In the subcloud layer, the profile looks more like that in the CBL.

The numerical grid in the stratocumulus case consists of $256^{3}$ points with a horizontal resolution of $\Delta x=\Delta y=25 \mathrm{~m}$ and a vertical resolution of $\Delta z=6.25 \mathrm{~m}$, spanning a domain of $6.4 \mathrm{~km} \times 6.4 \mathrm{~km} \times 1.6 \mathrm{~km}$. Like the smoke case, the kappa advection scheme for scalars and central-differences for momentum with a timestep of $\Delta t=0.5 \mathrm{~s}$ have been used. After 
two hours, regarded as spin-up period, the particles were released.

\subsubsection{Shallow cumulus topped boundary layer}

The shallow cumulus (in the remainder of the article referred to as cumulus) case used in this study is derived from the Small Cumulus Microphysics Study (SCMS) as described in Neggers et al. (2003). The cumulus topped boundary layer can be considered as two layers on top of each other. The subcloud layer has the characteristics of a dry CBL. The velocity distribution in the cloud layer is often thought and also parametrized (e.g., Siebesma and Cuijpers, 1995) as positively skewed: strong localized updrafts in the cloudy regions and homogeneously distributed compensating downdrafts elsewhere, see Fig. 1d. Recent studies by Heus and Jonker (2008) and Jonker et al. (2008) have however shown that downward mass transport occurs mainly near the edge of a cloud, a mechanism referred to as the subsiding shell.

From the buoyancy flux profile, Fig. 2d, it can be seen that cloud base is located at approximately $500 \mathrm{~m}$ and the cloud layer extends to $2500 \mathrm{~m}$. The subcloud layer has a profile similar to the CBL.

Numerical resolutions in the cumulus case are $\Delta x=\Delta y=25 \mathrm{~m}$ in the horizontal and $\Delta z=20 \mathrm{~m}$ in the vertical. With $256^{3}$ points, this amounts to a domain of $6.4 \mathrm{~km} \times 6.4 \mathrm{~km} \times 5.2 \mathrm{~km}$. A centered-difference integration scheme with a timestep of $\Delta t=1 \mathrm{~s}$ has been used. The particles were released after three hours of spin-up, allowing for a fully developed cumulus field.

\subsection{Scaling parameters}

In order to compare the results of the different boundary layers, we introduce the following dimensionless velocity and timescales. For the CBL, it would be natural to use the convective velocity scale based on the surface flux:

$w_{*}=\left(\frac{g}{\theta_{0}} \overline{\left(w^{\prime} \theta_{v}^{\prime}\right)_{0}} z_{i}\right)^{1 / 3}$

However in situations where the surface-flux is not the main driving mechanism of convection, an alternative velocity scale can be defined based on Deardorff (1980):

$w_{*}=\left(c_{1} \frac{g}{\theta_{0}} \int_{0}^{L_{z}} \overline{w^{\prime} \theta_{v}} d z\right)^{1 / 3}$

where $L_{z}$ is the domain height. The factor $c_{1}=2.5$ ensures that integrating a linear flux profile from a value of $\left(w^{\prime} \theta_{v}^{\prime}\right)_{0}$ at the surface to $0.2\left(w^{\prime} \theta_{v}^{\prime}\right)_{0}$ at the inversion height yields a velocity scale that is consistent with Eq. (1) . Equation (2) makes sense from a physical point of view, since the integral represents the production of turbulent kinetic energy. Furthermore, it is the most consistent choice, since we can now use Eq. (2) for all the cases under consideration. Although in the definition by Deardorff (1980) the integration is up to the inversion height $z_{i}$, we integrate over the entire domain because in the cumulus case the definition of the inversion height is not so clear. Concerning the other cases, since there is hardly any buoyancy flux above the inversion height, replacing $L_{z}$ by $z_{i}$ in Eq. (2) leads only to a very small difference.

The typical timescale is defined as

$t_{*}=\frac{z_{i}}{w_{*}}$.

\subsection{Statistics}

In this section we introduce the statistical variables necessary to describe the dispersion characteristics. The instantaneous local concentration $c(x, y, z, t)$ of particles is computed by counting the number of particles $N_{p}$ in a small control volume $\Delta V=\Delta x \Delta y \Delta z$ centered at $(x, y, z)$. This value is divided by the total number of particles $N_{\text {tot }}$ so that we have a normalized concentration:

$c(x, y, z, t)=\frac{N_{p}(x, y, z, t)}{N_{\text {tot }} \Delta x \Delta y \Delta z}$

$\int_{V} c d x d y d z=1$

The various statistical parameters can now be defined. The first statistical moment or mean plume height is given by

$\bar{z}=\int_{V} z c d x d y d z$

The vertical dispersion coefficient is defined by

$\sigma_{z}^{2}=\int_{V}(z-\bar{z})^{2} c d x d y d z$

where the difference with the mean plume height is used rather than the source height. For the skewness of the plume we get

$S_{z}=\frac{1}{\sigma_{z}^{3}} \int_{V}(z-\bar{z})^{3} c d x d y d z$

Another useful quantity is the horizontally integrated concentration, that we will call the vertical concentration profile

$C_{z}(z, t)=\frac{1}{A_{x y}} \int_{A} c d x d y$

were $A_{x y}=L_{x} L_{y}$ is the horizontal domain size. Equivalently we define a horizontal concentration profile according to

$C_{y}(y, t)=\frac{1}{A_{x z}} \int_{A_{x z}} c d x d z$

were $A_{x z}=L_{x} L_{z}$ is a vertical cross-section of the domain, $L_{z}$ denoting the domain height. 


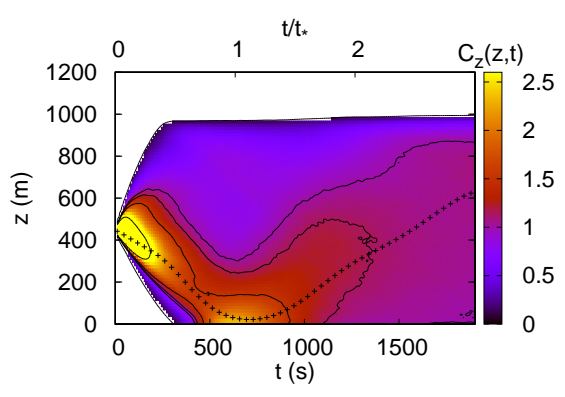

(a)

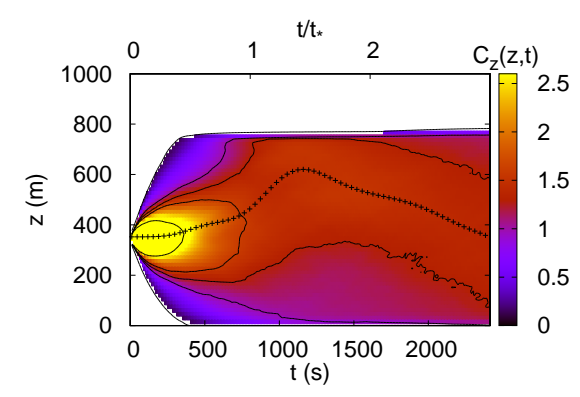

(c)

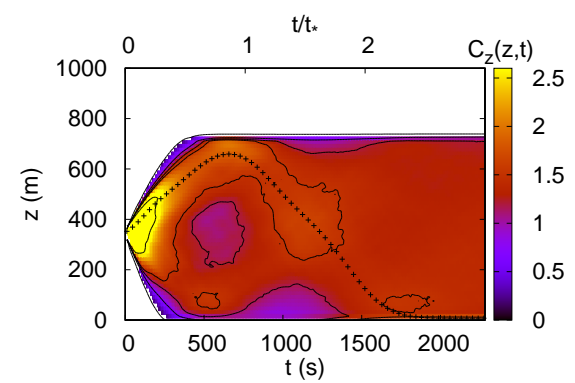

(b)

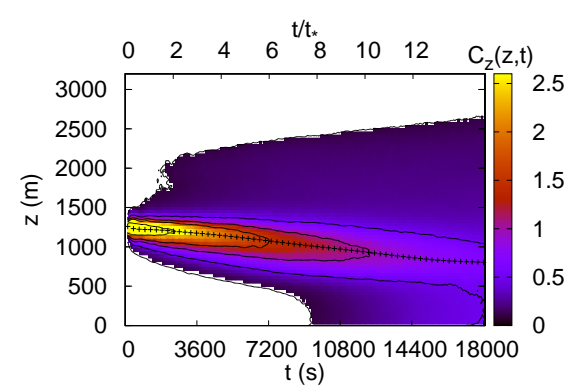

(d)

Fig. 3. Horizontally integrated plume evolution in four different boundary layers; (a) for the CBL, (b) for the smoke case, (c) for stratocumulus, and d) for cumulus. A plane of particles has been released instantaneously at half the boundary layer height and the evolution of the vertical concentration profile $C_{z}(z, t)$ as defined in Eq. (9) has been plotted. The concentration profile has been multiplied by 1000 to obtain a convenient scale. The points represent the location of the maximum concentration.

\subsection{Velocity statistics}

The probability density function (PDF) of velocity that we will consider is based on the velocities of the Lagrangian particles. Next to this PDF, we shall consider the Lagrangian velocity autocorrelation function, defined by

$R_{u}^{L}(\tau)=\frac{\overline{u^{\prime}(t) u^{\prime}(t+\tau)}}{\sigma_{u}^{2}}$

with $u^{\prime}(t)=u(t)-\overline{u(t)}$ the velocity fluctuation of a particle, the overbar represents the average over all particles at all times, and $u$ can be $u, v, w$.

\section{Results and discussion}

3.1 Plume phenomenology for different types of boundary layers.

To give a first general impression of the dispersion characteristics of the four different boundary layers, the time evolution of the concentration profiles, integrated over the two horizontal directions, are depicted in Fig. 3. For all the cases, $1024^{2} \approx 10^{6}$ particles were released instantaneously in a horizontal plane at half the boundary layer height, 2 or $3 \mathrm{~h}$, depending on the case, after the start of the simulation to allow for the spin-up. We briefly discuss the general features of Fig. 3. In the next section we will go into more detail for each case individually.

The evolution of the horizontally integrated plume in the CBL, Fig. 2a, has the familiar shape that was first described by Willis and Deardorff (1978): The plume concentration maximum initially is transported to the ground by the subsiding motions, then rises by the thermals, until eventually the plume is entirely mixed. After 3 turnover times the particles are almost homogeneously distributed throughout the boundary layer. The initial descent of the plume can be explained from the skewness of the vertical velocity distribution, as shown in Fig. 1a.

The plume evolution in the smoke cloud boundary layer resembles a reversed version of what happens in the dry $\mathrm{CBL}$. The plume maximum rises until it reaches the inversion, remains there some time and descends again. The observed plume can again be understood by considering the skewness of the vertical velocity distribution as schematically depicted in Fig. 1 b.

The plume evolution in the stratocumulus topped boundary layer is, at least for short times, much more symmetric than in the CBL and the smoke case. Making again the analogy with the previous cases, this can be explained by recalling that the stratocumulus topped boundary layer can be seen as a combination of the CBL and the smoke case. 


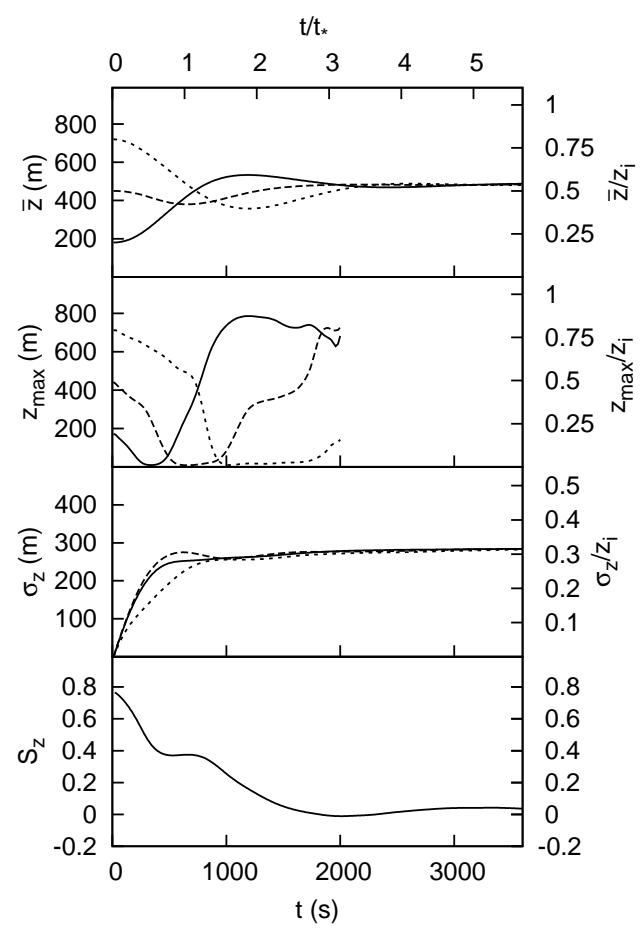

Fig. 4. Plume characteristics for the dry convective boundary layer. From top to bottom: mean plume height, height of maximum concentration level, dispersion coefficient for release from (solid line) $z=0.2 z_{i}$, (dashed line) $z=0.5 z_{i}$, and (dotted line) $z=0.8 z_{i}$; and skewness, for release from $z=0.5 z_{i}$.

Perhaps the most striking observation in Fig. 3 is the extremely slow plume spread in the shallow cumulus case, where the particles have been released at $1200 \mathrm{~m}$, i.e., in the middle of the cloud layer. The slow plume spreading is especially surprising given that the dispersion in the cloud layer, (Fig. 3d), is a composite of vigorously turbulent updrafts inside the clouds, and compensating motion in the environment. This configuration of the cloud layer results in a top-hat velocity distribution that is strongly skewed (Fig. 1d; Heus et al., 2009). On the basis of this velocity distribution, one would expect dispersion characteristics similar to those in the CBL. We will come back to this issue in Sect. 3.5.

\subsection{Statistics of dispersion in the CBL}

\subsubsection{Vertical dispersion}

The first, second and third order statistical moments and the height of maximum concentration as defined in the previous section have been plotted as a function of the dimensionless time in Fig. 4 for three different release heights.

The three different release heights $\left(0.2 z_{i}, 0.5 z_{i}\right.$ and $\left.0.8 z_{i}\right)$ have been chosen to cover a large part of the boundary layer, in order to observe how the dispersion characteristics change with height. The height of maximum concentration has not
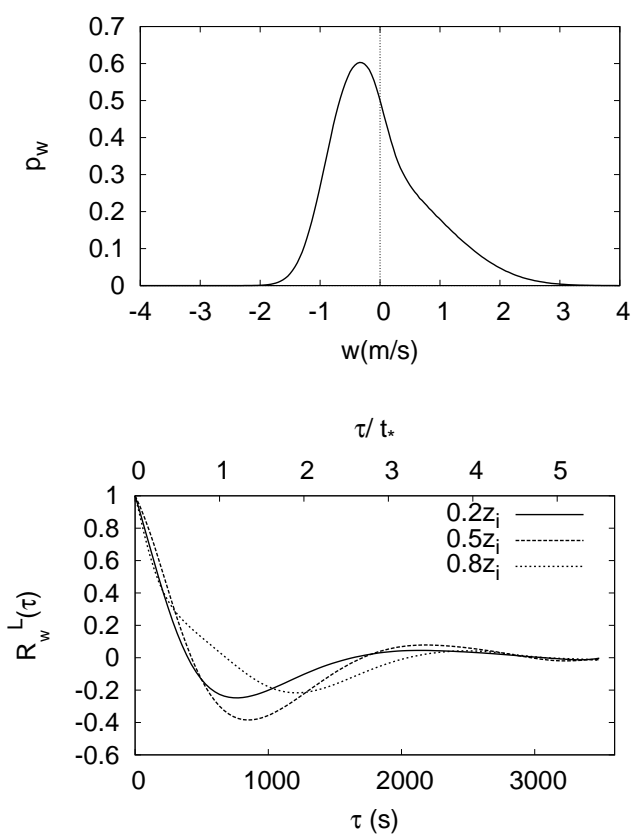

Fig. 5. Vertical velocity distribution based upon particles released homogeneously in the entire boundary layer and autocorrelation for different release levels in the CBL

been plotted for the full range, since this quantity becomes irrelevant when we approach a vertically homogeneous particle distribution. In the mean plume height and especially the location of the maximum we see the characteristics as described in the introduction: a near-ground release results in a steeply rising plume, whereas elevated release results in a descending plume. Initially, the skewness of the plume reflects the skewness in the vertical velocity distribution (only shown for release at $0.5 z_{i}$ ). A vertically homogeneous distribution is reached for all releases after approximately $t=4 t_{*}$. This well mixed situation is characterized by three conditions: the mean plume height is approximately half the boundary layer height $\bar{z} \approx 0.5 z_{i}$, secondly the vertical dispersion coefficient approaches the limit $\sigma_{z} / z_{i}=1 / \sqrt{12} \approx 0.3$ and finally the skewness of the plume should approach zero: $S_{z} \approx 0$. The results are in satisfactory agreement with other numerical studies by Nieuwstadt (1992) and Dosio and Vilà-Guerau de Arellano (2006), who in turn validated their results with experimental data from e.g. Willis and Deardorff (1978) and Briggs (1993).

\subsubsection{Vertical velocity statistics}

In Fig. 5 (top), the PDF of vertical velocity of the Lagrangian particles has been plotted and has indeed the positively skewed distribution. This PDF is based on particles released homogeneously in the entire CBL, so it represents the velocity distribution in the entire CBL rather than at a specific height. The Lagrangian autocorrelations of vertical 


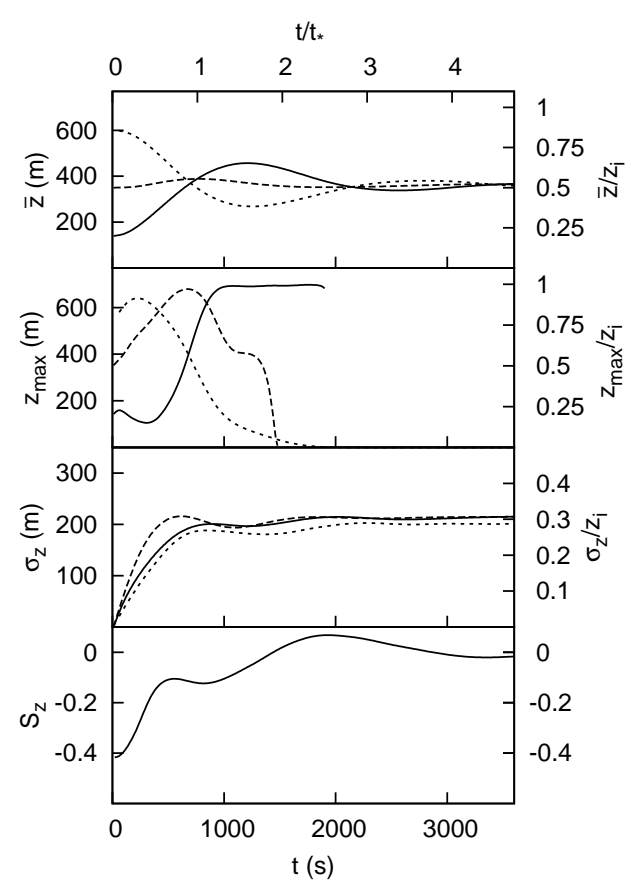

Fig. 6. Plume characteristics for the smoke case. From top to bottom: mean plume height, height of maximum concentration level, dispersion coefficient for release from (solid line) $z=0.2 z_{i}$, (dashed line) $z=0.5 z_{i}$, and (dotted line) $z=0.8 z_{i}$; and skewness, for release from $z=0.5 z_{i}$.

velocity for particles released at three different heights has been plotted in Fig. 5 (bottom). The autocorrelations are in agreement with the ones found by Dosio et al. (2005). The oscillating behaviour in the autocorrelation reflects the large scale coherent vertical motions.

We conclude that the dispersion results and the velocity statistics of the CBL are in satisfactory agreement with the literature. We shall therefore treat it as a reference case in understanding the results of the other cases.

3.3 Statistics of dispersion in the smoke cloud boundary layer

\subsubsection{Vertical dispersion}

In Fig. 6 the dispersion characteristics for the smoke cloud boundary layer are shown. As mentioned before, since the dynamics of the smoke cloud boundary layer can be regarded a mirror-image of the CBL, we see this directly in the dispersion characteristics. Considering the release at half the boundary layer height, where in the CBL the plume initially descends and impinges to the ground, in the smoke case the plume maximum rises until it reaches the capping inversion. Furthermore, we observe that a well-mixed distribution of particles $\left(\sigma_{z} \approx 0.3\right)$ is reached after approximately the same time as in the CBL. The skewness of the plume follows the
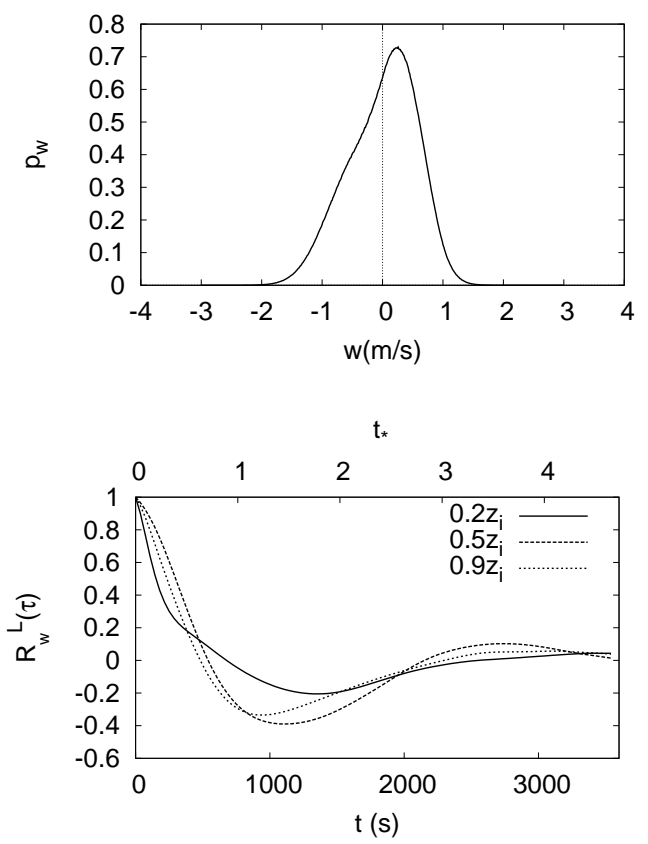

Fig. 7. Vertical velocity distribution and autocorrelation for the smoke case.

opposite behaviour as the one found for dispersion in the CBL, although it is somewhat smaller. The maximum concentration level for the low release height shows a small dip, before starting to rise as expected. Presumably because of the relatively small horizontal domain size of this case, events like penetrative up- and downdrafts show up immediately after particle release.

Another feature is the following: the mean plume height from the release at $0.5 z_{i}$ slightly ascends for a while, similarly but opposite to the mean plume height in the CBL. This might seem rather peculiar at first sight, since a mass balance over a horizontal plane is zero by conservation of mass, thus we would expect the mean plume height to remain constant. However, we must realise that the highest velocities are found in downdrafts and hence the particles that were initially in the strongest downdrafts already impinged to the ground while the majority of particles is still in a slow updraft halfway to the inversion. The fast moving descending particles can thus only "compensate" for the slow moving rising particles as long as they have not hit the ground yet. Indeed, a closer look shows that for very short times, the mean plume height is constant. The same phenomenon, although in the opposite direction, is observed for the mean plume height in the CBL case.

\subsubsection{Velocity statistics}

Figure 7 shows the vertical velocity distribution and the Lagrangian autocorrelation of vertical velocity in the smoke cloud boundary layer. As expected, we observe the reversed 
symmetry of the smoke case with the CBL, although the velocity distribution is somewhat narrower. The fact that we find here the same symmetry is not surprising, since the velocity statistics obviously determine the dispersion statistics. Concerning the autocorrelations, we observe again close agreement with the CBL. The autocorrelation becomes negative because of the coherent vertical motions that many particles undergo: they first reach the capping inversion where they cannot go any further and are then caught in a downdraft. It is worth noting in Fig. 7 that the line from the particles released closest to the ground that differs from the others, whereas in the CBL it is the line from the highest release that differs most. It seems that the autocorrelations of the velocity fields far away from the turbulent sources (the bottom in the CBL case, entrainment zone in the smoke case) are closer to the exponential function.

We conclude from the dispersion results in the smoke case that they can be understood in the light of the vertical velocity distribution in analogy with the CBL. Furthermore, also in the purely radiatively driven smoke case, i.e., in the absence of insolation to generate a surface heat flux, rapid mixing throughout the boundary layer is observed.

3.4 Statistics of dispersion in the stratocumulus topped boundary layer

\subsubsection{Vertical dispersion}

The statistical moments of the plume evolution in the stratocumulus case have been plotted in Fig. 8. We repeat here that to some extent the stratocumulus topped boundary layer can be regarded as a combination of the CBL and the smoke case, for it has both the surface flux characteristic for the CBL and radiative cooling at cloud top, like the smoke cloud. This is reflected partially in the dispersion characteristics, especially in the skewness of the plume, which is, although initially positive, much closer to zero than in the previous two cases, indicating a more symmetric plume evolution. The mean plume height and height of maximum concentration are more similar to the ones found in the smoke case though. Interestingly, a close inspection of Fig. 8 shows that for the release at $z=0.2 z_{i}$ the plume maximum descends to the ground before rising again and the release in the cloud layer does the opposite. This can possibly be explained by realising that the buoyancy flux profile in the subcloud layer is dominated by the positive surface flux (like the CBL), whereas the cloud layer looks more like the smoke case. de Roode and Duynkerke (1997) also noted that this leads to a skewness of the velocity distribution that changes with height: negatively skewed in the cloud layer and positively skewed in the subcloud layer. This means that dispersion around cloud base can be quite different depending on the exact location. Within the cloud the maximum concentration has a tendency to go up, but outside the cloud, the maximum will go down. In a well-coupled stratocumulus case like this one, the effect

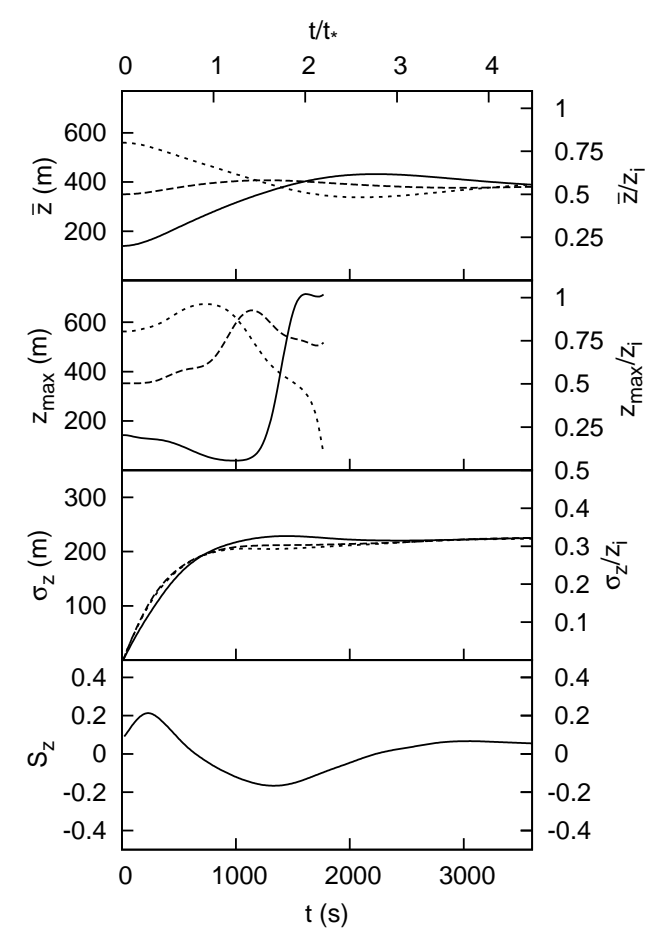

Fig. 8. Plume characteristics for the stratocumulus case. From top to bottom: mean plume height, height of maximum concentration level, dispersion coefficient for release from (solid line) $z=0.2 z_{i}$, (dashed line) $z=0.5 z_{i}$, and (dotted line) $z=0.8 z_{i}$; and skewness, for release from $z=0.5 z_{i}$.

is not very significant, but for a stronger decoupled stratocumulus case (i.e. the situation where there is a stably stratified layer just underneath cloud base, Duynkerke et al. (2004)) a clear separation between the "smoke-like" dispersion in the cloud layer and the "CBL-like" dispersion in the subcloud layer can be expected.

After $t \approx 3 t_{*}$ the particles are spread homogeneously in the vertical direction, comparable to the CBL and smoke case albeit a bit sooner than in the latter cases. This result confirms and quantifies the dispersion results for stratocumulus clouds by Sorbjan and Uliasz (1999). We emphasize again that this strong dispersion is contradicting simple dispersion models in which the dispersion parameter depends on the amount of insolation. A stratocumulus topped boundary layer has a cloud cover close to unity, and would have according to these models a dispersion coefficient almost an order of magnitude lower than in the CBL. Apparently, only considering the amount of insolation is insufficient to describe the dispersion in a cloudy boundary layer like the stratocumulus case.

\subsubsection{Velocity statistics}

In Fig. 9, the velocity statistics from the stratocumulus case have been plotted. The velocity distribution is much more symmetric than in the previous cases, which is in agreement 

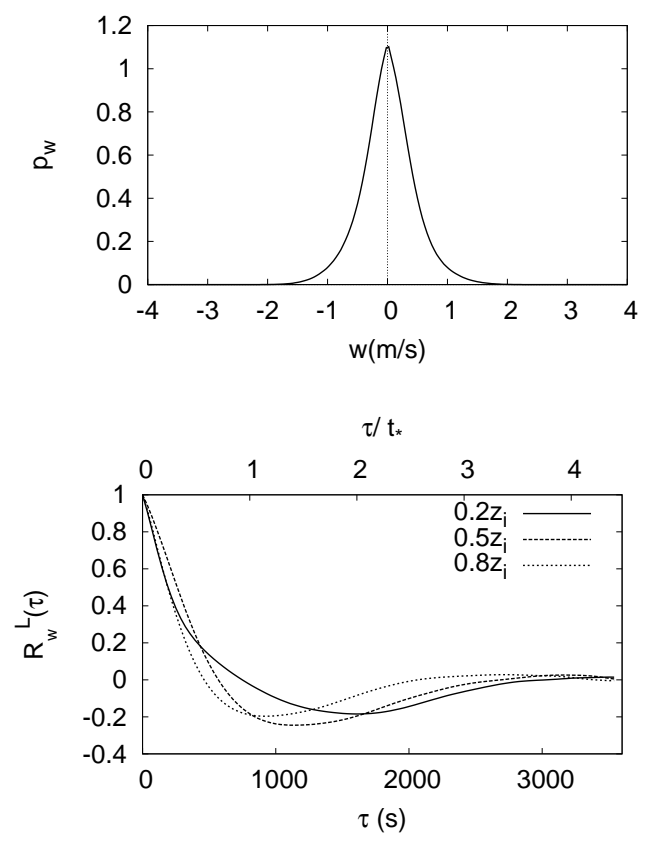

Fig. 9. Vertical velocity distribution and Lagrangian autocorrelation for the stratocumulus topped boundary layer.

with the characteristics of the plume. The autocorrelations very much resemble the ones from the CBL and the smoke case. The autocorrelation of the particles released at the top of the cloud layer has a shape similar to the one in the smoke case, again confirming that the stratocumulus cloud layer has the same characteristics as the smoke cloud.

Some final remarks about the generality of the results in the stratocumulus case. Stratocumulus clouds are found in many different forms; for example the decoupled situation, as mentioned above, can be expected to display different dispersion characteristics than the case we considered. The relative size of the cloud layer to the height of the boundary layer can also be expected to be of importance. Illustrative of the latter point are the results of Sorbjan and Uliasz (1999), who considered a stratocumulus case where the cloud layer covered almost the entire boundary layer and found dispersion results that were closer to the results of the smoke case from this study.

3.5 Statistics of dispersion in the cumulus topped boundary layer

\subsubsection{Vertical dispersion}

In the cumulus case it is important to distinguish between release in the sub-cloud layer and release in the cloud layer. Unless specified otherwise, the presented graphs represent the dynamics of the entire ABL, i.e. the composite over cloudy and clear regions.
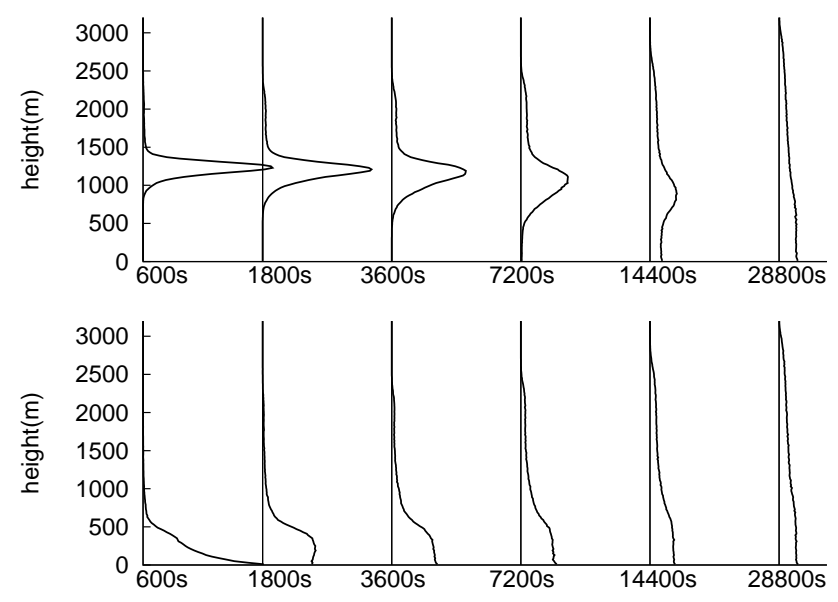

Fig. 10. Time evolution of the vertical concentration profile for the cumulus case. Bottom: release in the subcloud layer $(200 \mathrm{~m})$. Top: release in the cloud layer $(1250 \mathrm{~m})$.

From Fig. 2d it was already clear that dispersion in the cumulus cloud layer was very different than in the other cases. It is instructive to look at the plume evolution in another way than the contourplot from Fig. 3. Figure 10 shows the vertical concentration profile as defined by Eq. (9) for releases in the sub-cloud layer (bottom) and in the cloud-layer (top) at different times. Figure 11 shows the statistical moments of the plume evolution.

In Fig. 10 (bottom), it can be seen from the release in the sub-cloud layer that dispersion in the sub-cloud layer is initially analogous to the CBL: the plume maximum descends to the ground and then rises again. We also observe this in Fig. 11, although the location of the plume maximum has only been plotted for short times for release in the subcloud layer, because in a well mixed situation this quantity looses its relevance. After $30 \mathrm{~min}$ we observe a vertically well mixed profile in the sub-cloud layer, but clouds have transported a small part of the particles into the cloud layer. Clouds continue to bring particles upwards, so the concentration in the sub-cloud layer slowly decreases in time (cloud venting), whereas the number of particles in the cloud layer grows. These numerical results are in agreement with VilàGuerau de Arellano et al. (2005), although they did not observe the diminishing concentration in the subcloud layer since they prescribed a continuous surface flux of pollutants. The effect of cloud venting can also be seen in Fig. 11, where we see a steadily increasing dispersion coefficient for the release in the sub-cloud layer. One could speculate that this dispersion coefficient has two components: one from the dispersion in the sub-cloud layer, which is constant after approximately $30 \mathrm{~min}$ and one from the effect of cloud venting, which has a much larger time-scale.

Next we consider the release in the cloud layer. The plume is positively skewed, also observed in the bottom graph of Fig. 11, which reflects the skewed velocity distribution in 


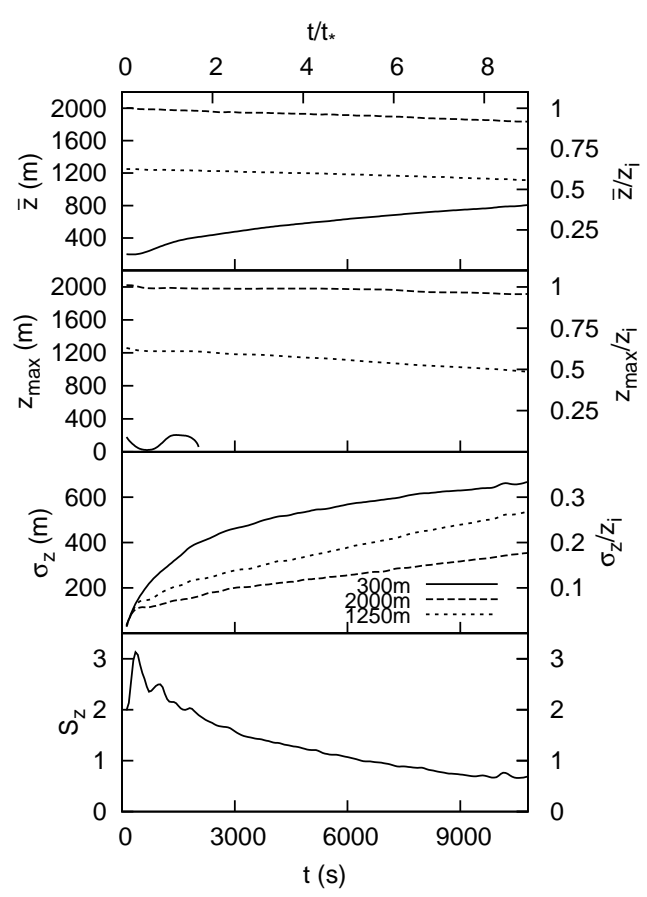

Fig. 11. Plume characteristics for the cumulus topped boundary layer. Note that cloudbase is approximately at $0.25 z_{i}$. From top to bottom: mean plume height, height of maximum concentration level, dispersion coefficient for release from (solid line) $z=0.2 z_{i}$, (dashed line) $z=0.5 z_{i}$, and (dotted line) $z=0.8 z_{i}$; and skewness, for release from $z=0.5 z_{i}$.

the cloud layer. The value of the skewness is about 3 times higher than in the dry CBL, which is due to the rare but very strong in-cloud updrafts in the cumulus layer (Heus et al., 2009). Nonetheless, looking at the height of the maximum concentration, we observe that it descends only very slowly, unlike we would expect from the analogy with the CBL. Moreover, the dispersion parameter shows that the plume spreads much slower in the cumulus cloud layer than in all other cases, not only in absolute sense (with the dispersion coefficient measured in meters), but also in dimensionless units. In the other cases, a vertically well mixed concentration profile was reached after $t \sim 3 t_{*}$; in the cumulus cloud layer this is not even the case after $t \sim 8 t_{*}$. The combination of both graphs in Fig. 10 provides the following conceptual picture: Clouds transport particles (pollutants) from ground level to the cloud layer, where they detrain from the cloud into the stable environment where in turn they hardly move anymore - a phenomenon sometimes referred to as plume trapping. We emphasize that this result is rather surprising and cannot be understood using the classical view on vertical transport by cumulus clouds, i.e., strong narrow updrafts in cloudy regions surrounded by homogeneously distributed downdrafts. The latter motions would transport the pollutants to cloud-base in a steady pace. The velocity statistics should clarify this issue.
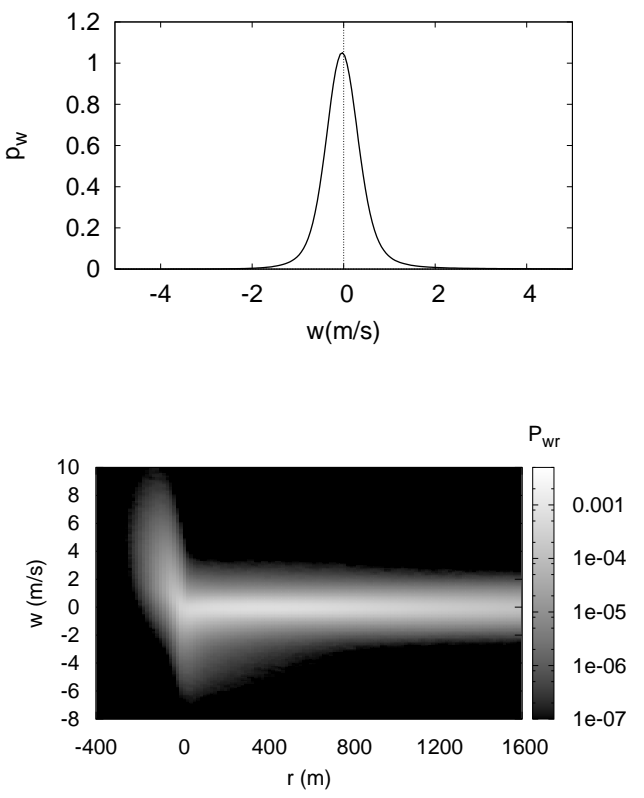

Fig. 12. Vertical velocity distribution in the cumulus cloud layer (top) and vertical velocity distribution as a function of distance to nearest cloud edge (bottom).

\subsubsection{Velocity statistics}

The distribution of vertical velocity in the cumulus cloud layer is shown in Fig. 12 together with a twodimensional velocity distribution, where the new coordinate $r$ represents the distance to the nearest cloud edge, following Jonker et al. (2008). Negative values for $r$ are locations inside the cloud. Jonker et al. (2008) and Heus and Jonker (2008) have shown that cumulus clouds are surrounded by a sheeth of descending air, resulting from evaporative cooling due to mixing with dry air outside the cloud. Figure 12 shows that indeed particles in a cloud move mostly upward, particles near a cloud move mostly downward. In the far environment particles have zero average velocity, although the variance of the velocity is clearly not zero. This velocity distribution explains why the plume maximum remains approximately constant, since the majority of the particles find themselves far away from clouds. However, this still does not explain why particles spread so slowly in the cumulus cloud layer, because, after all, the velocity distribution in the stratocumulus topped boundary layer is not so much different than in the cumulus cloud layer. To understand this we invoke the autocorrelation functions from Fig. 13. The autocorrelation of the particles released in the sub-cloud layer very much resembles the ones in the CBL, which is expected because the subcloud layer has all the characteristics of a CBL. For release in the cloudlayer, there seems to be a wavelike component in the autocorrelation function. Recalling that the cloud layer in a cumulus field is stably stratified for dry air, we can expect the presence of buoyancy waves, as is also shown in a theoretical 


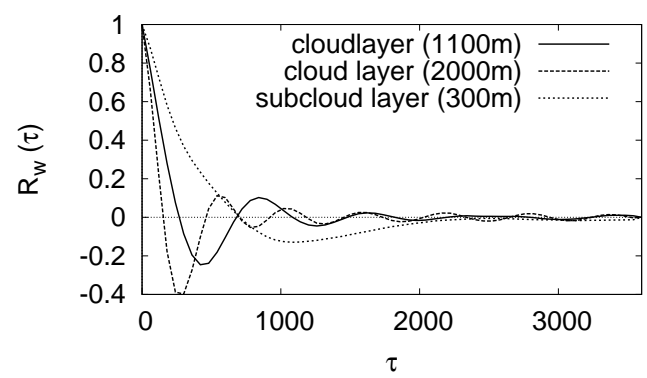

Fig. 13. Vertical velocity autocorrelation in the cumulus case.

analysis for stable boundary layers by Csanady (1973). The frequency of these oscillations, the Brunt-Väisälä frequency, is given by

$\omega=\sqrt{\frac{g}{\Theta_{0}} \frac{d \theta}{d z}}$

One could argue that the autocorrelation of the vertical velocity in a stratified medium is made of two components: one from stochastic motion associated with turbulence and one from the wavelike motion associated with buoyancy waves. Mathematically this would then translate to

$R^{L}(\tau)=e^{-\left(\frac{\tau}{T^{L}}\right)} \cos (\omega t)$

where $T^{L}$ is then some characteristic timescale over which the turbulent velocity is correlated. If the suggestion of buoyancy waves is indeed true, then $\omega$ in Eq. (13) should correspond with the one from Eq. (12). To verify this we computed the autocorrelations for many different release heights, fitted the results with Eq. (13) and so obtained a vertical profile of $T^{L}$ and $\omega$. From the LES fields we have the virtual temperature profiles, thereby allowing us to make a vertical profile of Eq. (12). Figure 14 shows the results. The values of $\omega$ as fitted from the autocorrelations correspond reasonably well with the ones calculated from the Brunt-Väisälä frequency. The difference between the two curves can be explained partially by realising that the autocorrelations represent ensembles of particles released at a certain height, but after a short while a part of these particles are not at their release height anymore.

The conceptual picture that emanates from the above considerations is that the vast majority of particles in the cumulus cloud layer is just lingering in the environment, every now and then get disturbed from their vertical position when a cloud perturbs the whole system and will then start to oscillate for a while around its original position. This explains why we do find a velocity distribution with a reasonable velocity variance, but yet hardly observe any plume spreading. The effective transport of pollutants is done solely by the cloud and the surrounding subsiding shell. Dispersion of pollutants released within a single cloud would first closely

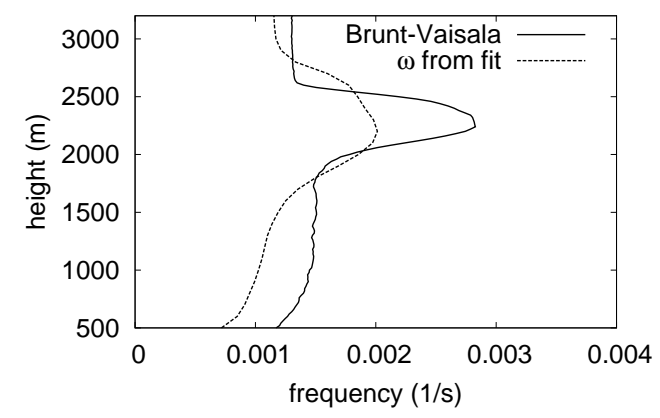

Fig. 14. Comparison between profiles of the Brunt-Väisälä frequency as calculated by Eq. (12) and the values of $\omega$ obtained by fitting Eq. (13) to the Lagrangian autocorrelations from different release heights.

resemble the cloud venting results as discussed by Chosson et al. (2008); after transporting the pollutants upward, the cloud disposes the pollutants in the stable cloud layer. The rather surprising conclusion from this view is that particles not only need a cloud to go up, but also one to go down in its surrounding shell.

\section{Conclusions}

We investigated the dispersion characteristics in different types of boundary layers in an LES based study together with a Lagrangian particle dispersion module. Comparison with the extensively documented dispersion in the dry convective boundary layer showed satisfactory agreement with the literature.

The vertical dispersion results from the smoke case and the stratocumulus case are well understood in terms of the vertical velocity distribution in the boundary layer. With the CBL as a reference case, the smoke case has the opposite velocity distribution and hence also mirrored dispersion characteristics. Radiative cooling at the top of the smoke cloud leads to strong narrow downdrafts and compensating updrafts. This negatively skewed velocity distribution translates into an initially rising plume maximum.

The stratocumulus case that we considered can best be viewed as combination of the smoke case and the CBL. The cloud layer, where radiative cooling is dominant, resembles the smoke case the most, whereas the sub-cloud layer, where the surface fluxes dominate, more resembles the CBL. A study of dispersion in other types of stratocumulus situations, such as the decoupled stratocumulus, would be interesting to pursue.

Future parametrizations of vertical dispersion in the smoke case and the stratocumulus case might benefit from exploiting the symmetry with respect to the CBL, where parametrizations have already been proposed and validated. 
It should be emphasized that in both the smoke case and the stratocumulus case we observe rapid mixing throughout the boundary layer. This observation contradicts simple dispersion models in which clouds have a damping effect on dispersion by blocking insolation. In addition, as already pointed out by Sorbjan and Uliasz (1999), stratocumulus can dramatically change the dynamics of the nocturnal boundary layer and corresponding dispersion characteristics.

The shallow cumulus case shows markedly different dispersion characteristics. Apart from the expected cloud venting (clouds transporting particles from the sub-cloud layer upwards), we observed that the dispersion in the cloud layer is much slower than in all other cases and as anticipated solely on the basis of the velocity distribution. It turns out that the velocity distribution as a function of distance to cloud and the vertical velocity autocorrelations need to be invoked to understand the observations. By doing so, the view that emanates is that particles far away from clouds display oscillating behaviour associated with buoyancy waves. This results in a velocity distribution with a variance comparable to the other three cases, but yet a very slow spreading of the plume. The overall picture that emerges is that clouds deposit pollutants emitted at ground level in the cloud layer, where they remain for very long times.

This view has consequences for the concentration variance in the cloud layer. Since pollutants in the environment far away from clouds mix very slowly throughout the cloud layer, there will remain areas with high concentrations for relatively long times. This might have consequences for chemical processes that are often non-linear with respect to the concentrations of the reactants. Additionally, this can be an important mechanism in establishing the initial conditions of the residual nocturnal boundary layer.

At a more detailed level than could be given in this study, many cloud characteristics have potentially significant influence on the dispersion in cloudy boundary layers. For instance, decoupling between the cloud layer and the subcloud layer, precipitation, shear and interaction with the free troposphere (especially for the stratocumulus case), and the precise configuration of the subsiding shell in the cumulus case, can alter the dispersion significantly. Such studies require in-depth studies of the respective cases, and could be interesting for future work. However, we believe that, like in the dry CBL, a reliable first order view has been given here by considering the strength, amount and location of the convective updrafts and downdrafts.

Acknowledgements. This work was sponsored by the Stichting Nationale Computerfaciliteiten (National Computing Facilities Foundation, NCF), with financial support from the Nederlandse Organisatie voor Wetenschappelijk Onderzoek (Netherlands Organization for Scientific Research, NWO).

Edited by: S. Galmarini

\section{References}

Angevine, W.: An integrated turbulence scheme for the boundary layer with shallow cumulus aplied to pollutant transport, J. Appl. Meteor., 44, 1436-1452, doi:10.1175/JAM2284.1, 2005.

Bretherton, C. S., MacVean, M. K., Bechtold, P., Chlond, A., Cuxart, J., Khairoutdinov, M., Kosovic, B., Lewellen, D. C., Moeng, C.-H., Siebesma, A. P., Stevens, B., Stevens, D. E., I.Sykes, and Wyant, M. C.: An intercomparison radiatively-driven entrainment and turbulence in a smoke cloud, as simulated by different numerical models, Q. J. Roy. Meteor. Soc., 125, 391-423, 1999.

Briggs, G.: Plume Dispersion in the Convective Boundary Layer. Part II: Analyses of CONDORS Field Experiment Data, J. Appl. Meteorol., 32, 1388-1425, 1993.

Ching, J. K. S., Shipley, S. T., and Browell, E. V.: Evidence for cloud venting of mixed layer ozone and aerosols, Atmos. Environ., 22, 225-242, 1988.

Chosson, F., Paoli, R., and Cuenot, B.: Ship plume dispersion rates in convective boundary layers for chemistry models, Atmos. Chem. Phys., 8, 4841-4853, 2008, http://www.atmos-chem-phys.net/8/4841/2008/.

Cotton, W.: Cloud Venting - A review and some new global annual estimates, Earth-Sci. Rev., 39, 169-206, 1995.

Csanady, G.: Turbulent diffusion in the environment, D. Reidel Publishing company, 1973.

Cuijpers, J. W. M. and Duynkerke, P. G.: Large-eddy simulation of trade wind cumulus clouds, J. Atmos. Sci., 50, 3894-3908, 1993.

de Roode, S. and Duynkerke, P.: Observed Lagrangian transition of stratocumulus into cumulus during ASTEX: mean state and turbulence structure, J. Atmos. Sci., 54, 2157-2173, 1997.

Deardorff, J. W.: Stratocumulus-capped mixed layers derived from a three-dimensional model, Bound.-Lay. Meteorol., 18, 495-527, 1980.

Dosio, A. and Vilà-Guerau de Arellano, J.: Statistics of Absolute and Relative Dispersion in the Atmospheric Convective Boundary Layer: A Large-Eddy Simulation Study, J. Atmos. Sci., 63, 1253-1272, 2006.

Dosio, A., Vilà-Guerau de Arellano, J., Holtslag, A. A. M., and Builtjes, P. J. H.: Relating Eulerian and Lagrangian statistics for the turbulent dispersion in the atmospheric convective boundary layer, J. Atmos. Sci., 62, 1175-1191, 2005.

Duynkerke, P. G., de Roode, S. R., and coauthors: Observations and numerical simulation of the diurnal cycle of the EUROCS stratocumulus case, Q. J. Roy. Meteor. Soc., 140, 3269-3296, 2004.

Heus, T. and Jonker, H. J. J.: Subsiding shells around shallow cumulus clouds, J. Atmos. Sci., 65, 1003-1018, 2008.

Heus, T., van Dijk, G., Jonker, H. J. J., and Van den Akker, H. E. A.: Mixing in Shallow Cumulus Clouds Studied by Lagrangian Particle Tracking, J. Atmos. Sci., 65, 2581-2597, 2008.

Heus, T., Pols, C. F. J., Jonker, H. J. J., Van den Akker, H. E. A., and Lenschow, D. H.: Observational validation of the compensating mass flux through the shell around cumulus clouds, Q. J. Roy. Meteor. Soc., 135, 101-112, doi:10.1256/qj.08.66, 2009.

Hundsdorfer, W., Koren, B., Vanloon, M., and Verwer, J. G.: A positive finite-difference advection scheme, J. Comput. Phys., 117, 35-46, 1995.

Hunt, J.: Diffusion in the Stably Stratified Atmospheric Boundary Layer, J. Appl. Meteorol., 24, 1187-1195, 1985. 
Jonker, H. J. J., Heus, T., and Sullivan, P. P.: A refined view of vertical transport by cumulus convection, Geophys. Res. Lett., 35, L07810, doi:10.1029/2007GL032606, 2008.

Kemp, J. R. and Thomson, D. J.: Dispersion in stable boundary layers using large-eddy simulation, Atmos. Environ., 30, 2911-2923, http://www. sciencedirect.com/science/article/B6VH3-3Y45YPP-C/\%2/ bbc713b160704afb7bcdc444fcabeb58, 1996.

Lamb, R. G.: A numerical simulation of dispersion from an elevated point source in the convective planetary boundary layer, Atmos. Environ., 12, 1297-1304, 1978.

Neggers, R. A. J., Jonker, H. J. J., and Siebesma, A. P.: Size statistics of cumulus cloud populations in large-eddy simulations, J. Atmos. Sci., 60, 1060-1074, 2003.

Nieuwstadt, F. and de Valk, J.: A large eddy simulation of buoyant and non-buoyant plume dispersion in the atmospheric boundary layer, Atmos. Environ., 21, 2573-2587, 1987.

Nieuwstadt, F. T. M.: A large-eddy simulation of a line source in a convective atmospheric boundary layer-I. Dispersion characteristics, Atmos. Environ. Part A. General Topics, 26, 485-495, 1992.

Pasquill, F.: The estimation of the dispersion of windborne material, Meteor. Mag., 90, 33-49, 1961.

Siebesma, A. P. and Cuijpers, J. W. M.: Evaluation of parametric assumptions for shallow cumulus convection, J. Atmos. Sci., 52, 650-666, 1995.

Sorbjan, Z. and Uliasz, M.: Large-Eddy Simulation of air Pollution Dispersion in the Nocturnal Cloud-Topped Atmospheric Boundary Layer, Bound.-Lay. Meteorol., 91, 145-157, 1999.
Taylor, G.: Diffusion by continuous movements., Proc. London Math. Soc., 20, 196-212, 1921.

Thomson, D. J.: Criteria for the selection of stochastic models of particle trajectories in turbulent flows, J. Fluid Mech., 180, 529556, 1987.

Vilà-Guerau de Arellano, J., Kim, S.-W., Barth, M. C., and Patton, E. G.: Transport and chemical transformations influenced by shallow cumulus over land, Atmos. Chem. Phys., 5, 32193231, 2005, http://www.atmos-chem-phys.net/5/3219/2005/.

Weil, J., Patton, E., and Sullivan, P.: Lagrangian modeling of dispersion in the stable boundary layer, in: 17th Symposium on Boundary Layers and Turbulence, 2006.

Weil, J. C., Lawson, R. P., and Rodi, A. R.: Relative Dispersion of Ice Crystals in Seeded Cumuli, J. Appl. Meteorol., 32, 10551073, 1993.

Weil, J. C., Sullivan, P. P., and Moeng, C. H.: The use of large-eddy simulations in Lagrangian particle dispersion models, J. Atmos. Sci., 61, 2877-2887, 2004.

Wicker, L. J. and Skamarock, W. C.: Time-Splitting Methods for Elastic Models Using Forward Time Schemes, Mon. Weather Rev., 130, 2088-2097, 2002.

Willis, G. E. and Deardorff, J. W.: A laboratory study of dispersion from an elevated source within a modeled convective planetary boundary layer, Atmos. Environ., 12, 1305-1311, 1978. 